\title{
Hybrid Approach for Localization in Anisotropic Sensor Networks
}

\author{
King-Yip Cheng, King-Shan Lui and Vincent Tam \\ Department of Electrical and Electronic Engineering \\ The University of Hong Kong \\ Pokfulam Road, Hong Kong, China
}

\begin{abstract}
In many real-world applications including agricultural, meterological, military applications, etc, localization techniques are widely used to estimate the geographic locations of sensor nodes based on the precision positions of a few anchors equipped with special hardware. Existing localization algorithms mainly try to improve their accuracy in position estimation by using various heuristic-based or mathematical techniques. Every node in the network follows the same technique to find its physical location. However, each individual method with its own strength can only outperform the others in some but not all nodes. Based on this observation, we develop a hybrid approach for the localization problem. Each node collects the same kind of information. By analysing the information, a node can decide what is the best localization algorithm to use. Different nodes can make their own decisions. Our simulation results reveal that the hybrid approach is effective that it outpeforms existing algorithms. To the best of our knowledge, our work presents the first effort in solving the absolute localization problem by adopting a hybrid approach.
\end{abstract}

\section{INTRODUCTION}

A wireless sensor network [1], [2], [3] is a network consisting of thousands of sensors that span a large geographical region. These sensors are able to communicate with each other to collaboratively detect objects, collect information, and transmit messages.

In many applications, sensors have to know their geographical locations. Theoretically, the Global Positioning System (GPS) can be used for a sensor to locate itself. In reality, it is not practical to use the GPS in every sensor node because a sensor network consists of thousands of nodes and the GPS becomes very costly. To solve the problem, many localization methods have been developed. Instead of requiring every node to have the GPS installed, all localization methods [4], [5], [6] assume only a few nodes $(\geq 3)$ are equipped with the GPS hardware. These nodes are called anchor or beacon nodes that know their positions without communicating with other nodes. Other normal sensors then obtain distance information through talking to each other and derive their positions based on the information.

Most of the existing work focus on increasing the accuracy in position estimation by using different mathematical techniques such as triangulation [3], multilateration, multidimensional scaling (MDS) [1], [5], proximity mapping (PDM) [2], convex optimization [7], etc. In all the above methods, the same algorithm is applied throughout the whole sensor network. However, algorithms do not perform equally well throughout the network, for example, the method in [7] is more accurate if sensors are within the convex hull ${ }^{1}$ formed by beacons. Besides, performance is also affected by the network topology. For example, MDS-MAP [8], [5] has better performance in uniform topologies while PDM [2] outperforms MDS-MAP in anisotropic ${ }^{2}$ topologies. The strengths of different algorithms under different scenarios demonstrate that a hybrid approach combining these algorithms may yield a better performance in terms of the overall accuracy.

We perform simulations to study whether a single algorithm always outperforms the others in all nodes of a sensor network. We focus on anisotropic networks since generally speaking, anisotropic sensor networks possess challenging properties to many localization algorithms due to various limiting factors including the geographical shape of the involved region, different node densities, irregular radio patterns, etc. We study PDM [2] and the improved APS ${ }^{3}$. We select PDM to study because it is the best distributed localization algorithm in the literature. Improved APS is also studied because APS is one of the pioneer work to the problem. We generate 30 different networks, each of 250 nodes with $10 \%$ as beacons. Among 225 nodes that require localization, on average, 125 nodes find more accurate positions through the improved APS when the radio range is 1 unit. In other words, about half of the nodes can get better position information through PDM while another half can compute their locations more accurately through the improved APS. When the radio range is increased, the percentage of nodes that the improved APS performs better also increases. When the radio range is 2 unit, the improved APS outperforms PDM in about $85 \%$ of the nodes.

Our simulation results is a convincing evidence that nodes in a network should not always use the same localization algorithm. To reduce errors in position estimation, each node should decide which algorithm to use depending on its situation. In this paper, we describe a simple but yet effective method for a node to decide whether PDM or the improved APS should be used. We evaluate our protocol through simu-

\footnotetext{
${ }^{1}$ A convex hull of a set of points is the minimum convex polygon that contains all the points in the set

${ }^{2} \mathrm{~A}$ network topology is isotropic if the properties of proximity measures are identical in all directions; otherwise, the network is anisotropic. One of the most representative anisotropic topologies is the C-shape topology.

${ }^{3}$ In the improved APS, a non-anchor node tries to find "good" anchor nodes for position estimation
} 
lations. The results reveal our hybrid approach outperforming the original approach of using PDM or improved APS alone. This paper is organized as follows. Section II discusses some related works. In Section III, we explain our simple yet efficient convex hull detection algorithm (CHDM) in detail, and describe how a node uses CHDM to determine which localization algorithm to use. Section IV gives the simulation results of our proposed hybrid algorithm and compare them against the PDM and APS. Lastly, we conclude our work in Section V.

\section{RELATED WORKS}

Niculescu and Nath [3] propose the Ad-hoc Positioning System (APS) that extends the capabilities of the well-known Global Positioning System (GPS) [10] to non-GPS enabled nodes in a hop-by-hop manner in an ad hoc network including any wireless sensor network. APS is one of the earliest methods developed for localization. It is based on the triangulation used in GPS and is a distributed protocol that requires reasonable computational memory and message overheads. APS assumes there are at least three anchor nodes, nodes that know their exact physical locations, in a sensor network. Each normal sensor tries to find out its distance to the anchor nodes. When the distance information to three or more anchor nodes is obtained, the sensor node can compute its own position using triangulation. The key question that APS answers is how a node finds out its distances to the anchors. Three methods are described: DV-hop, DV-distance, and "Euclidean" propagation. Among them, DV-hop and DVdistance receive most attention. Both DV-hop and DV-distance measure distance in a hop-by-hop manner.

One source of error in APS is the estimation of the real physical distance between a node and an anchor is not accurate. DV-distance is measuring the path distance between a node and an anchor. The difference between the path distance and the physical distance can be very large in an anisotropic network. For example, in the C-shape network shown in Figure 1 , the real distance between the nodes marked as a star is less than 7.07 but the path distance between them is larger than 19.31 .

In view of this, Lim and Hou [2] take the difference between real distance and path distance into consideration. Anchor nodes measure their path distances with each other. Since they all have exact location information, they also can compute their physical distances. A proximity-matrix is then derived from the distances to illstrate how to map a path distance to the corresponding physical distance. This matrix is distributed to every node in the network. When a node measures its path distance to a certain anchor, it can use the matrix to get a better estimate of the real physical distance from itself to the anchor node. Two types of proxmities can be used to determine the proximity vector, i.e hop count or estimated geographic distance. When hop count is used, the operation is similar to DV-hop of APS. When estimated geographic distance is used, the operation is similar to DV-distance of APS. Localization system based on PDM is also distributed. It should be noted

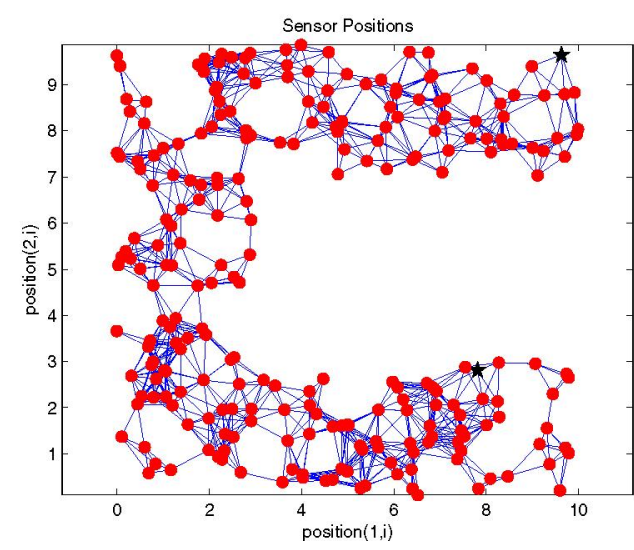

Fig. 1. C-Shape Network

that PDM, basically as the transformation matrix, precisely characterizes anisotropic network topologies since it retains the components of proximities to the anchor nodes in all directions.

Both APS and PDM are distributed algorithms. Centralized algorithms have also been developed by researchers to tackle the localization problem. Shang et al. [8] consider a centralized 3-step method, namely the MDS-MAP. The original MDSMAP algorithm first uses an all-pair shortest-path algorithm to give a roughly estimated distance between all possible pairs of nodes. Then, the algorithm proceeds to apply the multidimensional scaling (MDS) to derive node locations fitting the initially estimated distances. An immediate result is a global and relative map of all nodes involved. Lastly, with the absolute positions of 3 or more anchors, the relative map can be transformed into the absolute coordinates of all the sensor nodes. Extensive simulations reveal that the MDSMAP performs particularly well in cases with few anchors and relatively uniform distributions of nodes. However, with the initial requirement for computing all-pairs shortest-paths distance, the original MDS-MAP suffers from the drawbacks as being a computationally expensive and centralized algorithm. In view of this problem, Shang et al. [5] later propose a new variant, namely the patched MDS-MAP (MDS-MAP(P)), that supports distributed computing environment and also performs exceptionally well in irregular topologies. The main idea is to build a small relative local map for each individual node. The relative maps can then be merged together to form a global map that will be further improved by a global refinement step to minimize the estimation errors. As compared to the original MDS-MAP, the improved MDS-MAP(P) algorithm is much more computationally expensive. However, the MDS-MAP(P) remarkably outperforms most existing methods for irregular topologies with a relatively small number of anchors available.

Ahmed et al. [11] propose a hybrid approach SHARP, combining APS and MDS to do relative localization. The localization process is divided into two phases. In phase 1, some sensor nodes are selected as reference nodes and a relative map of the reference nodes is built using MDS. In 
phase 2, the reference nodes are considered as anchors and APS is used to localize the non-reference nodes relative to the coordinate system constructed by the reference nodes. SHARP outperforms MDS in relative localization in terms of the Performance-Cost Metric (PCM). However, the two-phase approach may pose a synchronization problem. Nodes have to be syncronized so that non-reference nodes know exactly when to start phase 2 after phase 1 is completed.

Localization problem is also formulated into different kinds of optimization problems. Doherty et al. [12], Biswas et al. [13], Camillo[14] formulate it into convex programming, semidefinite programming and linear programming, respectively. Although algorithms are provided in [12], [13],[14] to solve the optimization problems, they are centralized in nature. The complexity and scalability may become issues when these algorithms are deployed in real sensor networks.

\section{HYBRID APPROACH: PDM+CHDM}

Since a sensor network may consist of thousands of nodes, a truly distributed approach is more appropriate. Therefore, we focus our studies on distributed algorithms APS and PDM.

As mentioned in Section I, we study whether a single algorithm outperforms the others in all nodes of a sensor network. Our results show that neither APS nor PDM can be an absolute winner. APS performs better for some nodes while PDM performs better for the others. If a node can smartly identify which method is better, the position estimation can be more accurate.

We thus develop an algorithm for each non-anchor node to determine whether APS or PDM should be used. Nodes obtain distance information according to the original PDM protocol. Then, a condition is checked and if the condition is satisfied, APS is used; otherwise, PDM is used. The detection mechanism is called Convex Hull Detection Method (CHDM) and it has the following desirable features:

\section{- Little Overhead}

CHDM is based on the same information that APS works on, that is, the path distances to at least 3 anchor nodes and the positions of those anchor nodes. Thus, combining CHDM and PDM does not require any extra control messages but only some computational overheads to execute CHDM. As CHDM requires only several comparisons of distance measurements per node, the complexity of the hybrid approach is virtually the same as PDM. Due to the fact that the energy needed for communication is much larger than the energy required for computation, the extra energy spent in CHDM is negligible.

- Distributed

Each node executes the same detection algorithm and makes its own decision independently. No synchronization is needed. On the other hand, decisions do not have to be distributed to neighbors or any base stations. The algorithm does not make assumption on the underlying topology and can be used in networks of different sizes and shapes.
We now describe the Convex Hull Detection Method in detail. The aim of the detection method is to identify nodes that are more suitable to use APS for deriving locations. In other words, we should identify conditions where APS can perform better. Through analysis and extensive simulations, we have identified several factors affecting the performance of APS as follows:

- Number of anchors used

Our previous work [9] shows that using all anchors in a network to derive locations may not always yield the most accurate results. The accuracy of APS can be improved by considering only a suitable subset of anchor nodes, say the nearest 3 anchors to each node, rather than using all anchor nodes when applying to anisotropic sensor networks.

- Positions of anchor nodes

Due to the nature of triangulation, when 3 anchors are used and the non-anchor node is outside the triangle formed by the anchors, position estimation is not very accurate.

- Distances of anchor nodes

As mentioned in Section II, if the path distance differs largely from the physical distance, APS cannot give accurate results. If the anchor node is only one hop away, the path distance should be much the same as the physical distance. Intuitively, the difference between the path distance and the physical distance should be related to the number of hops between the two nodes. When more hops are encountered in estimating the distance, the estimation tends to be more erroneous, especially in anisotropic networks. The fewer the hops, the better the APS performance.

The CHDM is based on the above factors. For a non-anchor node $x, \mathrm{CHDM}$ is used to check whether there are 3 anchor nodes among all anchors available to likely form a convex hull embedding $x$. If a convex hull can be formed and all 3 anchors are within $k$ hops from $x$, the position of $x$ is calculated through triangulation with the anchors forming the convex hull. The $k$-hop requirement tries to avoid the path distance deviating a lot from the real physical distance. If no convex hull can be found, or the hop count from any one of the anchors forming the convex hull is larger than $k$, the node of interests will use PDM to calculate its position. Determining whether $x$ is more than $k$ hops away from an anchor is trivial since this information is provided by the APS or PDM advertisements. In contrast, convex hull detection is more difficult since $x$ does not know its physical location. We now describe our heuristic in solving the problem.

Let the 3 concerned anchor nodes be $a 1, a 2$, and $a 3$. CHDM considers the distances of $x$ to $a 1, a 2$, and $a 3$ and the respective positions of the three anchors. To facilitate our discussion, let the line connecting $a 1$ and $a 2$ be $e 12$, and $a 2$ and $a 3$ be $e 23$, etc. The line connecting $x$, which is assumed to be inside the convex hull at the moment, to anchor node $a 1$ is $l 1$ accordingly. Figure 2 shows the spatial relationship 
of the involved anchors and node $x$.

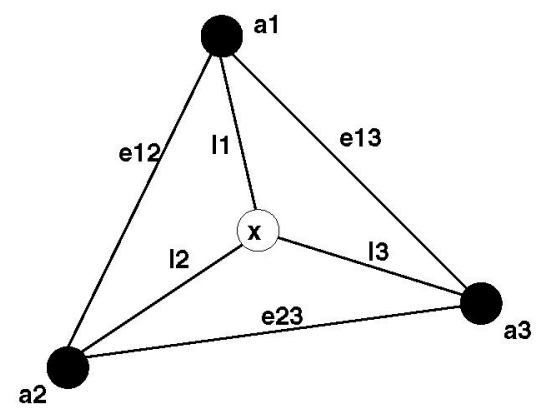

Fig. 2. The spatial relationship of all involved anchors and node $x$

Clearly, the following properties should hold:

$$
\begin{aligned}
& l 1<e 12 \text { and } l 1<e 13 \\
& l 2<e 12 \text { and } l 2<e 23 \\
& l 3<e 13 \text { and } l 3<e 23
\end{aligned}
$$

where $<$ means shorter in length. It is true that the above simple condition also holds for some points that lie outside the convex hull. But it should be able to identify those points that are far outside the convex hull.

\section{Simulation}

To evaluate the performance of the hybrid approach, simulations are conducted using MatLab Version 7.04. We generate 30 different $\mathrm{C}$-shape topologies, each of 250 nodes with 25 anchors. 250 nodes are first randomly placed in the C-shape area and then 25 nodes are randomly chosen to be anchors. Linearized lateration algorithm [3] is used. $k$ is set to 4 . Communication range $R$ is set from $1 r$ to $2 r$ which results in different connectivity. Table I gives the average connectivity (average number of neighbors) with different range $R$. The position error of each node is normalized by the range $R$ which is calculated as follows:

$$
\text { error }=\frac{\left\|x_{i}^{\prime}-x_{i}\right\|}{R}
$$

$x_{i}$ and $x_{i}^{\prime}$ are the true and the estimated position of node $i$ respectively. $\|*\|$ denotes the Euclidean norm.

We compare our algorithm against the original PDM and APS with CHDM improvement. In APS with CHDM improvement, nodes that can pass the CHDM test find their positions using the anchors that form a convex hull. Other nodes that cannot find a convex hull simply choose three nearest anchors for triangulation. Figure 3 shows the average error of each approach. For all ranges selected, the hybrid approach outperforms the original PDM and APS with CHDM improvement. Although APS with CHDM improvement does not perform very well when compared with PDM, especially when the range is small, our hybrid approach successfully identifies nodes where APS with CHDM are better and reduces the average error.

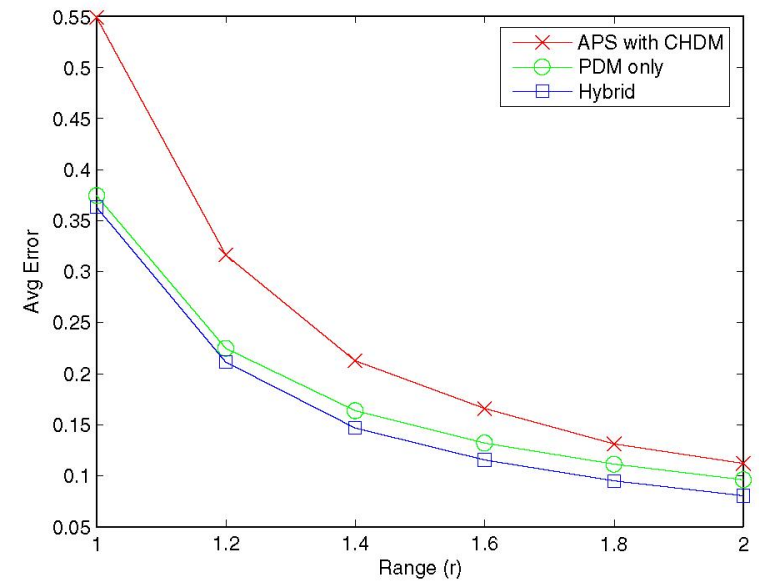

Fig. 3. Average Position Error with $n=250, m=25$

\begin{tabular}{|c||c|}
\hline Range & Connectivity \\
\hline $1 r$ & 9.17 \\
\hline $1.2 r$ & 12.72 \\
\hline $1.4 r$ & 16.68 \\
\hline $1.6 r$ & 21.01 \\
\hline $1.8 r$ & 25.63 \\
\hline $2 r$ & 30.38 \\
\hline
\end{tabular}

TABLE I

AVERAGE CONNECTIVITY FOR DIFFERENT RADIO RANGES

\section{CONClusion}

In this paper, we demonstrate that a single localization algorithm should not be used throughout the network as algorithms perform differently under different conditions. Localization algorithm should be chosen dynamically by each node. We propse a hybrid approach using APS and PDM. Each nonanchor node chooses the localization method based on the result of convex hull detection method (CHDM) and the hop count between the node of interests and the anchors. Simulation results show that the hybrid approach outperforms the original approaches in different radio ranges.

In most real-world applications, wireless sensor networks would consist of various local regions with different proximity measures and local properties possibly due to geographical or other physical limitations, i.e. mostly anisotropic. Therefore, it makes sense to provide an adaptive hybrid that can flexibly apply different localization methods according to the network topologies or local properties measured.

Our future works is to investigate the feasibility of incorporating more approaches like MDS to make the hybrid approach more flexible and adaptive.

\section{REFERENCES}

[1] X. Ji and H. Zha, "Sensor positioning in wireless sensor networks using multidimensional scaling," in IEEE Infocom, 2004.

[2] H. Lim and J. C. Hou, "Localization for anisotropic sensor networks," in IEEE Infocom, 2005. 
[3] D. Niculescu and B. Nath, "Ad hoc positioning system (aps)," in IEEE Globecom, 2001, pp. 2926 - 2931

[4] J. Hightower and G. Boriello, "Location systems for ubiquitous computing," in IEEE Computer, vol. 34, no. 8, August 2001, pp. 57 - 66.

[5] Y. Shang and W. Ruml, "Improved mds-based localization," in IEEE Infocom, 2004.

[6] C. Savarese, J. Rabaey, and K. Langendoen, "Robust positioning algorithm for distributed ad-hoc wireless sensor networks," in USENIX Technical Annual Conference, 2002.

[7] L. Doherty, K. Pister, and L. Ghaoui, "Convex position estimation in wireless sensor networks," in IEEE Infocom, 2001.

[8] Y. Shang, W. Ruml, and Y. Zhang, "Localization from mere connectivity," in ACM MobiHoc, 2003.

[9] K. Y. Cheng, V. Tam, and K. S. Lui, "Improving APS with anchor selection in anisotropic sensor networks," in Proceedings of the ICNS'05, 2005 .

[10] B. P. et al., "Global positioning system: Theory and application : Vol. i," in Progress in Astronautics and Aeronautics, Volume 163, 1996.

[11] A. A. Ahmed, H. Shi, and Y. Shang, "Sharp: A new approach to relative localization in wireless sensor networks," in IEEE ICDCS, 2005.

[12] L. Doherty, K. S. J. Pister, and L. Ghaoui, "Convex position estimation in wireless sensor networks," in IEEE Infocom, 2001.

[13] P. Biswas and Y. Ye, "Semidefinite programming for ad hoc wireless sensor network localization," in IEEE IPSN, 2004

[14] C. Gentile, "Sensor location through linear programming with triangle inequality constraints," in IEEE ICC, 2005. 